\title{
Tecnura
}

http://revistas.udistrital.edu.co/ojs/index.php/Tecnura/issue/view/640

DOI: http://dx.doi.org/10.14483/udistrital.jour.tecnura.2014.SE1.a01

INVESTIGACIÓN

\section{Problema de tomografía local usando wavelets B-spline cúbicos}

\author{
Local tomography problem using cubic B-spline wavelets
}

\author{
Wilmar Alberto Díaz Ossa*, Harold Vacca González**
}

Citation / Para citar este artículo: Diaz Ossa, W., \& Vacca González, H. (2014). Problema de tomografía local usando wavelets B-spline cúbicos. Revista Tecnura, Edición especial, 13-29.

Fecha de recepción: 14 de febrero de 2013 / Fecha de aceptación: 7 de marzo de 2014

\section{RESUMEN}

En el presente artículo se describe e implementa una solución al problema de la tomografía local, equivalente a la inversión de la transformada de Radon, utilizando la transformada wavelet. Para ello se ejecuta un algoritmo basado en wavelets B-spline cúbicos de soporte compacto con suficientes momentos de desvanecimiento para que la función de escalado filtrada, la wavelet madre y su transformada de Hilbert tengan decaimiento rápido. Lo anterior favorece la localización de la transformada wavelet de la transformada de Radon y, por tanto, la inversión, es decir; la reconstrucción de una región central de interés del fantasma Shepp-Logan.

Palabras clave: Base separable biortogonal, B-Spline cúbico, tomografía local, transformada de Radon, wavelets.

\begin{abstract}
This paper presents a formal description and subsequent implementation of a solution to the local tomography problem, equivalent to the so-called Radon Transform local Inversion. To do so, it is necessary to execute an algorithm based on compact-support B-spline cubic wavelets, including sufficient vanishing moments so that the filtered-scaling, the mother wavelet and its Hilbert Transform functions decay rapidly. This favors localization of Radon-Transform Wavelet Transform and, therefore, its corresponding inversion; that is, the reconstruction of a Shepp-Logan Phantom central interest region (ROI).
\end{abstract}

Keywords: Separable biorthogonal base, cubic B-spline, local tomography, Radon transformation, wavelets.

\footnotetext{
* Licenciado en matemáticas, Universidad Pedagógica Nacional; magíster en matemáticas aplicadas, Universidad EAFIT. Docente de la Universidad Distrital Francisco José de Caldas, Bogotá, Colombia. Contacto: wadiazo@udistrital.edu.co

** Licenciado en matemáticas, Universidad Distrital Francisco José de Caldas; magíster en matemáticas aplicadas, Universidad EAFIT. Docente de la Universidad Distrital Francisco José de Caldas, Bogotá, Colombia. Contacto: hvacca@udistrital.edu.co
} 


\section{INTRODUCCIÓN}

En los problemas inversos, el objetivo es estimar algunos atributos desconocidos que son de interés, a partir de mediciones que se relacionan indirectamente con dichos atributos. Tal es el caso de la tomografía, en donde, a partir de las mediciones de las atenuaciones de los rayos $X$ que atraviesan un objeto, se debe recuperar la imagen del mismo. Este asunto empezó a investigarse teóricamente hacia 1917 por J. Radon; posteriormente, hacia 1979, Cormack y Hounsfield concretaron sus resultados en la tomografía axial computarizada; y desde finales del siglo XX hasta hoy, el problema ha seguido vigente por efectos del avance en tecnología computacional y la variedad de aplicaciones que pueden desarrollarse.

El artículo se estructura de la siguiente manera: en la sección 2 se describe el problema de la tomografía y, en particular, el de la tomografía local o de región de interés; en la sección 3 se presentan la notación y las definiciones de las transformadas utilizadas; en la sección 4 se hace una descripción de los antecedentes y métodos utilizados en la reconstrucción de una función a partir de la transformada de Radon; en la sección 5 se definen las transformadas wavelets; en la sección 6 se hace una introducción al análisis wavelet que incluye el análisis multirresolución para determinar las wavelet escalada y madre, y se utilizan wavelets B-spline biortogonales como alternativa para recuperar la transformada de Radon. En la sección 7 se estudian las wavelets para invertir Radon; en la sección 8 se muestran y analizan los resultados obtenidos al comparar la imagen de la región central del fantasma Shepp-Logan, reconstruida al utilizar wavelets separables biortogonales, frente a la reconstrucción realizada utilizando transformada rápida de Fourier.

Finalmente, en la sección 9 se presentan las conclusiones.

\section{EL PROBLEMA DE LA TOMOGRAFÍA LOCAL}

Al hacer pasar un número finito de rayos $X$ a través de un plano de un objeto, desde varios ángulos, se busca cómo construir el mapa de intensidades del objeto a partir de las medidas de las atenuaciones de los rayos. Para ello se considera una variable $s$, que mide la distancia desde la fuente a lo largo de un rayo, asumiéndose que la intensidad / cambia con respecto a tal distancia. Faridani, Keinert, Natterer, Ritman \& Smith (1990); Faridani, Ritman \& Smith (1993); Faridani, Finch, Ritman \& Smith (1997) habían encontrado que la relación entre la intensidad inicial transmitida $\left(I_{0}\right)$, la cantidad de materia (densidad, $\mu(x, y))$, e intensidad final $\left(I_{T}\right)$, está dada por:

$$
\frac{d I}{I}=-\mu(x, y) d s
$$

Donde

$$
\frac{I_{0}}{I_{T}}=e^{-\int_{\sigma} \mu(x, y) d s}
$$

Berenstein \& Walnut (1994 y 1995), así como Rashid-Farrokhi, Liu, Berenstein \& Walnut (1994), indicaban que el problema de la tomografía local se presenta como la recuperación de la función $f(x) \chi_{\{|x| \leq a\}}(x)$, dado $a>0$, a partir de las proyecciones $\mathfrak{R}_{\theta} f(s) \chi_{[-a, a]}(s)$; donde $\chi_{s}(t)$ es la función característica y $\mathfrak{R}_{\theta} f(s)$ es la transformada de Radon de $f$. La idea se centra en la inversión de la transformada de Radon, es decir: teniendo todas las proyecciones el objetivo consiste en la recuperación de la función. Walnut (1993); Rashid-Farrokhi et al. (1994); o más recientemente J. Walker (2008), indicaban que la tomografía local también se denomina problema interior, o tomografía en una región de interés (ROI, por sus siglas en inglés). Maass (1992); Helgason (1999); Natterer (2001) y Natterer \& Wübbeling (2001), enunciaban además que la inversión de la transformada de Radon no es única en dimensiones pares. Esto quiere decir que el 
problema de la tomografía local no tiene solución única cuando $n$ es par.

\section{DEFINICIONES Y NOTACIÓN}

Los conceptos dados a continuación se definen y notan, al estilo de Berenstein \& Walnut (1994 y 1995); Mertins (1996); Folland (1999); Natterer (2001); Pinsky (2001); Hong, Wang \& Gardner (2005); Boggess \& Narcowich (2007); Chaudhury \& Unser (2011), entre otros.

$L_{1}(R)$ es el espacio de todas las funciones $f: R \rightarrow C$, tal que $\int_{R}|f(t)| d t=\|f\|_{L_{1}}<\infty$. De igual forma, se tiene para $L_{2}(R)$, el espacio de las funciones cuadrado-integrables, con la norma definida a partir de la ecuación (3):

$$
\|f\|_{L_{2}}=\left(\int_{R}|f(t)|^{2} d t\right)^{1 / 2}<\infty .
$$

Dadas $f \in L_{1}\left(\mathrm{R}^{n}\right) \quad$ y $\quad \xi=\left(\xi_{1}, \xi_{2}, \ldots, \xi_{n}\right)$, $x=\left(x_{1}, x_{2}, \ldots, x_{n}\right) \in \mathrm{R}^{n}$, se define la transformada de Fourier de $f$ en el punto $\xi$ mediante (4).

$$
\hat{f}(\xi)=\frac{1}{(2 \pi)^{\frac{n}{2}}} \int_{\mathbb{R}^{n}} f(x) e^{-i(\xi, x)} d x
$$

Donde $\quad\langle\xi, x\rangle=\xi_{1} x_{1}+\xi_{2} x_{2}+\ldots+\xi_{n} x_{n} \quad y$ $d x=d x_{1} d x_{2} \ldots d x_{n}$

La transformada de Hilbert se define a partir de la ecuación (5).

$$
H f(t)=f_{H}(t)=\frac{1}{\pi} \int_{\mathrm{R}} \frac{f(\tau)}{t-\tau} d \tau
$$

La transformada inversa de Hilbert está dada por (6).

$$
f(t)=\frac{1}{\pi} \int_{\mathrm{R}} \frac{f_{H}(\tau)}{t-\tau} d \tau
$$

$S^{n-1}$, define la esfera unidad; sea $\Theta$ que pertenece a $S^{n-1}, \theta^{\perp}$ es el hiperplano ortogonal a $\Theta$ que pasa a través del origen.
La transformada de Radon de una función $f(x)$, $x \in \mathrm{R}^{n}$ está definida por la ecuación (7).

$$
\mathfrak{R} f(\theta, s)=\mathfrak{R}_{\theta} f(s)=\int_{\vec{x} \cdot \vec{\theta}=s} f(\vec{x}) d \vec{x}=\int_{\theta^{\perp}} f(s \vec{\theta}+y) d y
$$

$\Re f(\theta, s)$ representa la integral de línea de $f$, en el hiperplano de $\mathrm{R}^{n}$ perpendicular a $\Theta$ y a una distancia $s$ desde el origen. Dada una función $h(\theta, t)$ definida en $S^{n-1} \times \mathrm{R}$, se define el operador retroproyección $\mathfrak{R}^{\#}$ a partir de la ecuación (8).

$$
\mathfrak{R}^{\#} h(x)=\int_{S^{n !}} h(\theta, x \cdot \theta) d \theta
$$

Donde el par $(\theta, t)$ es la parametrización del hiperplano $\{x: x \cdot \theta=t\}$, y la función $h$ está definida en el hiperplano $(n-1)$-dimensional de $\mathrm{R}^{n}$. En este caso, $\mathfrak{R}^{\#} h(x)$ es la integral de $h$ sobre todos los hiperplanos que pasan a través de $x$.

Por otra parte, dados $m \geq 0, p \geq 1$ y $\Omega$ un dominio de $\mathrm{R}^{n}(n \geq 2)$; el espacio de Sobolev, $W^{m, p}(\Omega)$ se define mediante (9).

$$
W^{m, p}(\Omega)=\left\{u \in L_{p}(\Omega): \partial^{\alpha} u \in L_{p}(\Omega), \quad \forall \alpha,|\alpha| \leq m\right\} .
$$

$Y$ se dice un espacio vectorial normado, equipado con la norma expresada en (10).

$$
\|u\|_{W^{m, p}(\Omega)}=\left(\int_{\Omega} \sum_{|\alpha| \leq m}\left|\partial^{\alpha} u\right|^{p} d x\right)^{1 / p}=\left(\sum_{|\alpha| \leq m}\left\|\partial^{\alpha} u\right\|_{L_{p}(\Omega)}^{p}\right)^{1 / p}
$$

\section{METODOLOGÍA Y ANTECEDENTES}

Guédon \& Unser (1992); Ramm \& Zaslavsky (1993); Berenstein \& Walnut (1994 y 1995), así como Rashid-Farrokhi, Liu, Berenstein \& Walnut (1994); Natterer (2001) y Natterer \& Wübbeling (2001), entre otros, coinciden en que la metodología más utilizada para invertir la transformada de Radon, se basa en el teorema de Slice de Fourier, ecuación (11). Dada $f(x), x \in \mathrm{R}^{n}$ y $\theta \in S^{n-1}$ fijo

$$
\mathfrak{R}_{\theta} \hat{f}(\lambda)=\hat{f}(\gamma \theta)
$$


Para todo $\gamma \in \hat{R}$ ( $\hat{R}$ representa el dual de $R$ ).

$\mathrm{Al}$ escribir la fórmula usual de inversión de Fourier en coordenadas polares se tiene la ecuación (12).

$$
f(x)=\int_{S_{+}^{n \perp 1}} \int_{-\infty}^{\theta} \mathfrak{R}_{\theta} \hat{f}(r) e^{2 \pi i((x \theta) r)}|r|^{n-1} d r d \theta
$$

Donde $S_{+}^{n-1}$ denota la mitad superior de la esfera en $\mathrm{R}^{n}$. Berenstein \& Walnut (1994 y 1995); Natterer (2001), observan que la ecuación (12) puede generalizarse de la siguiente manera. Dado $\alpha \in R$, se define el operador potencial de Riesz (ecuación (13)), I $I^{\alpha}$ como:

$$
I^{\alpha} \hat{f}(\xi)=|\xi|^{-\alpha} \hat{f}(\xi)
$$

Si $\alpha<n$, y $g=\mathfrak{R}_{\theta} f$ entonces se obtiene la ecuación (14).

$$
\begin{gathered}
f=\frac{1}{2}(2 \pi)^{1-n} I^{-\alpha} R^{\#} I^{\alpha-n+1} g \\
f=c_{n}\left\{\begin{array}{cc}
R^{\#} H g^{(n-1)}, & \text { n par } \\
R^{\#} g^{(n-1)}, & \text { nimpar }
\end{array}, \quad c_{n}=\frac{1}{2}(2 \pi)^{1-n} \begin{cases}(-1)^{(n-2) / 2} & \text { n par } \\
(-1)^{(n-1) / 2} & \text { nimpar }\end{cases} \right.
\end{gathered}
$$

Esto significa que la fórmula de la inversión es local en el siguiente sentido: para reconstruir $f$ en algún punto $x$, solo se necesita la integral de $f$ sobre hiperplanos que pasan a través de un entorno de $x$.

Un segundo método para invertir la transformada de Radon es a través de la fórmula de retroproyección filtrada (ecuación (15)). Dado $f(x)$, $x \in \mathrm{R}^{n}, g(\theta, t)=g_{\theta}(t), \theta \in S^{n-1}, t \in \mathrm{R}$,

$$
f * \mathfrak{R}^{\#}\left(g_{\theta}\right)(x)=\mathfrak{R}^{\#}\left(\mathfrak{R}_{\theta} f * g_{\theta}\right)(x)
$$

La convolución del lado izquierdo se calcula con respecto a $x \in \mathrm{R}^{n}$ y la derecha con respecto a $t \in \mathrm{R}$. Guédon y Unser (1992) utilizaron este método para calcular los cuadrados de las proyecciones de funciones polinómicas suaves por tramos, con nodos espaciados uniformemente.

Natterer (2001), Delaney \& Bresler (1995) o Destefano \& Olson (1994), formalmente utilizan para la reconstrucción de $f$, entre otras, la ecuación (16).

$$
f(x)=\frac{1}{4 \pi^{2}} \int_{0}^{2 \pi} \int_{\mathrm{R}} \frac{\frac{d}{d s} g(\theta, s)}{(x \cdot \theta)-s} d s d \theta
$$

Esta, sin embargo, es una forma difícil de implementar computacionalmente.

\section{TRANSFORMADAS WAVELETS}

El origen de la descomposición de una señal en wavelets radicó en la necesidad de conocer las características y particularidades de la señal en diferentes instantes de tiempo. Muchos autores han actuado en consecuencia; por ejemplo, Mallat (1989, 1992 y 2008); Daubechies (1990, 1992 y 2006); Chui (1992); Aldroubi (1996); Hernández \& Weiss (1996); Goswami \& Chan (1999); Boggess \& Narcowich (2007), observaron que el análisis de Fourier tiene el defecto de la no localidad, es decir, el comportamiento de una función en un conjunto abierto, sin importar cuán pequeño, influye en el comportamiento global de la transformada de Fourier; por lo que no se captan los aspectos locales de la señal tales como cambios bruscos, saltos o picos, que se han de determinar a partir de su reconstrucción. Este aspecto se soluciona a través del análisis wavelet.

\section{Transformada wavelet continua}

En particular, Mallat (1989, 1992 y 2008) y Daubechies (1990, 1992 y 2006), construyen la teoría wavelet basada en la representación de una función en términos de una familia biparamétrica de dilataciones y traslaciones de una función fija $\psi$, Ilamada wavelet madre.

Definición 5.1. Para $f, \psi \in L_{2}(\mathrm{R})$, la transformada wavelet se expresa mediante la ecuación (17).

$$
W_{\psi} f(a, b):=\int_{\mathrm{R}} f(t) \overline{\psi_{a, b}(t)} d t
$$

Por la desigualdad de Cauchy, $W_{\psi}$ es una función acotada: $\left|W_{\psi} f(a, b)\right| \leq\|f\|_{2}\|\psi\|_{2}$. Nótese también que la transformada se puede escribir como la ecuación (18). 


$$
W_{\psi} f(a, b)=\left\langle f, \psi_{a, b}\right\rangle_{L_{2}(\mathrm{R})}=\left\langle f, \psi_{a, b}\right\rangle
$$

Luego, la transformada wavelet $W_{\psi}$ de $f$ puede ser descrita en términos de convolución: $W_{\psi} f(a, b)=\left(f \cdot \hat{\psi}_{a, 0}\right)(b)$ donde $\hat{\psi}(t)=\overline{\psi(-t)}$. Además: $\hat{\widetilde{\psi}}_{a, b}(\omega)=\sqrt{|a| \hat{\psi}}(a \omega) e^{-i \omega b}$.

La fórmula de inversión, en términos de $W_{\psi}$, está dada por la ecuación (19); se puede ver, por ejemplo, Mallat (1989) y Mallat \& Hwang (1992).

$$
f(t)=\frac{1}{C_{\psi}} \int_{\mathrm{R}^{2}} W_{\psi} f(a, b) \psi_{a, b}(t) d b \frac{d a}{a^{2}}
$$

\section{Transformada wavelet discreta}

Daubechies (1990, 1992 y 2006), Mallat (1989), entre otros, consideran que la transformada wavelet continua introduce cierta redundancia, pues la señal original se puede reconstruir completamente calculando $W_{\psi} f(a, \cdot)$ para una cantidad numerable de escalas, por ejemplo, potencias enteras de 2. Esto es, si se elige la escala $a=2^{-j}$ para cada $j \in \mathbf{Z}$, y también se discretiza en el dominio del tiempo en los puntos $b=2^{-j} k, k \in Z$, la familia de wavelets será ahora dada por la ecuación (20).

$$
\psi_{2^{-j}, 2^{-j} k}(t)=\frac{1}{\sqrt{2^{-j}}} \psi\left(\frac{t-2^{-j} k}{2^{-j}}\right)=2^{j / 2} \psi\left(2^{j} t-k\right), \quad \forall j, k \in Z .(20)
$$

Se utilizará la notación $\psi_{j, k}$ para denotar la wavelet $\psi$ comprimida $2^{j}$ y trasladada $k$ unidades, es decir, $\psi_{j, k}(t)=2^{j / 2} \psi\left(2^{j} t-k\right)$. Con la elección de $a=2^{-j}$ y $b=2^{-j} k$, el muestreo en el tiempo se ajusta proporcionalmente a la escala; es decir, a mayor escala se toma puntos más distantes, ya que se busca información global, mientras que a menor escala se buscan detalles de la señal, por tal motivo se muestrea en puntos menos distantes entre sí. Para otras elecciones de $a$ y $b$ se puede consultar a Chui (1992). En consecuencia, una condición suficiente para la reconstrucción de una señal $f$ es que la familia de dilatadas y trasladadas $\psi_{j, k}$ forme una base ortonormal en el espacio $L_{2}(\mathrm{R})$. Para conocer más detalles se pueden ver Hernández \& Weiss (1996) y Daubechies (1990). Si esto se tiene, cualquier función $f \in L_{2}(\mathrm{R})$ se puede escribir mediante la ecuación (21).

$$
f(t)=\sum_{j, k} c_{j, k} \psi_{j k}(t)
$$

Donde $c_{j, k}=\left\langle f, \psi_{2^{-j}, 2^{-j} k}\right\rangle=W_{\psi} f\left(2^{-j}, 2^{-j} k\right)$.

Definición 5.2: Para cada $f \in L_{2}(\mathrm{R})$, el conjunto bidimensional de coeficientes se determina mediante la ecuación (22).

$$
c_{j, k}=\left\langle f, \psi_{j k}\right\rangle=\int_{\mathrm{R}} 2^{j / 2} f(t) \overline{\psi\left(2^{j} t-k\right)} d t
$$

Se denomina la transformada wavelet discreta de $f$.

En consecuencia, la expresión (serie-wavelet) se puede escribir en forma alterna como la ecuación (23).

$$
f(t)=\sum_{j, k}\left\langle f(t), \psi_{j k}(t)\right\rangle \psi_{j k}(t)
$$

La serie expresada en la ecuación (23) se llama representación wavelet de $f$.

Para recuperar $f(t)$ desde su transformada wavelet, $\psi(t)$ debe satisfacer la ecuación (24).

$$
\hat{\psi}(0)=\int_{-\infty}^{\infty} \psi(t) d t=0
$$

Además, las wavelets se construyen de modo que tengan soporte compacto y un alto orden de desvanecimiento. Una wavelet se dice que tiene momentos de desvanecimiento de orden $m$ si cumple la ecuación (25).

$$
\int_{\mathrm{R}} t^{p} \psi(t) d t=0, \quad p=0,1, \ldots, m-1
$$

La expresión para la transformada wavelet inversa se define mediante la ecuación (26),

$$
f(t)=\frac{1}{C_{\psi}} \int_{R} d b \int_{0}^{\infty} \frac{1}{a^{2}}\left[W_{\psi} f(b, a) \psi_{b, a}(t)\right] d a
$$


la cual permite reconstruir la función original desde su transformada wavelet. Aquí, $C_{\psi}$ es una constante que depende de la elección de la wavelet y se conoce como condición de admisibilidad, y se define a partir de la ecuación (27).

$$
C_{\psi}:=\int_{\mathrm{R}} \frac{|\hat{\psi}(\omega)|^{2}}{|\omega|} d \omega<\infty
$$

$\hat{\psi}(\omega)$ es la transformada de Fourier de $\psi$.

Si además $\psi \in L_{1}(\mathrm{R})$, entonces la condición (13) implica que $\int_{R} \psi(t) d t=0$. En efecto, por el lema de Riemann-Lebesgue (ver, por ejemplo: Meyer (1990); Irino \& Kawahara (1993); Wojtaszczyk (1997) o Walnut (2002)), $\lim _{\omega \rightarrow \infty} \hat{\psi}(\omega)=0$, y dada la continuidad de la transformada de Fourier, implica que $0=\hat{\psi}(0)=\int_{\mathrm{R}} \psi(t) d t$.

\section{Transformada rápida wavelet}

Rioul \& Duhamel (1992), así como Destefano \& Olson (1994) o Walter \& Shen (1999), determinan que la transformada rápida wavelet es una implementación computacionalmente eficiente de la transformada wavelet discreta, que aprovecha la relación entre los coeficientes de la transformada wavelet discreta y escalas adyacentes, lo que se expresa en las ecuaciones (28) y (29).

$$
\begin{aligned}
& W_{\psi}(j, k)=\sum_{m} h_{\psi}(m-2 k) W_{\phi}(j+1, m) \\
& Y \\
& W_{\phi}(j, k)=\sum_{m} h_{\phi}(m-2 k) W_{\phi}(j+1, m)
\end{aligned}
$$

Estas ecuaciones pueden expresarse mediante convolución (ecuaciones (30) y (31)), con los coeficientes wavelet y de escalamiento invertidos en el tiempo (descomposición), es decir,

$$
\begin{aligned}
& W_{\psi}(j, k)=\left.h_{\psi}(-n) * W_{\phi}(j+1, m)\right|_{n=2 k, k \geq 0} \\
& \mathrm{Y} \\
& W_{\phi}(j, k)=\left.h_{\phi}(-n) * W_{\phi}(j+1, m)\right|_{n=2 k, k \geq 0}
\end{aligned}
$$

Donde las convoluciones son evaluadas en instantes $n=2 k$ para $k \geq 0$.

\section{ANÁLISIS MULTIRRESOLUCIÓN Y FUNCIONES SPLINE}

El concepto de análisis multirresolución fue desarrollado por Mallat (1989) y Meyer (1990). Aplicando este tipo de análisis a una función esta puede descomponerse en funciones más simples y estudiarlas separadamente, núcleo fundamental del concepto siguiente:

Definición 6. Un análisis multirresolución (AMR) sobre $\mathrm{R}$ consta de una sucesión de subespacios cerrados $\left\{V_{j}: j \in Z\right\}$ de $L^{2}(\mathrm{R})$, que satisfacen:

1. $V_{j} \subset V_{j-1} ; \quad \forall j \in Z$ (cadena de subespacios encajonados).

2. $\overline{\cup_{j \in Z} V_{j}}=L^{2}(\mathrm{R})$, es decir $\cup_{j \in Z} V_{j}$ es densa en $L^{2}(\mathrm{R})$.

3. $\cap_{j \in Z} V_{j}=\{0\}$.

4. $f(x) \in V_{j}$ si y solo si $f\left(2^{j} x\right) \in V_{0}$.

5. $f(x) \in V_{0}$ si y solo si $f(x-k) \in V_{0}$, para todo $k \in Z$, además, por (4) se tiene que si $f(x) \in V_{j}$, entonces $f\left(x-2^{j} k\right) \in V_{j}$, para todo $k \in Z$.

6. Existe $\phi \in V_{0}$ tal que $\left\{\phi_{0, k}: k \in Z\right\}$ es una base ortonormal $V_{0}$.

Las condiciones (1)-(3) significan que cualquier función en $L_{2}(\mathrm{R})$ se puede aproximar por elementos de los sub-espacios $V_{j}$, y como $j$ tiende a $\infty$ la precisión de la aproximación se incrementa. Las condiciones (4) y (5) expresan la invariancia de la familia de sub-espacios $\left(V_{j}\right)_{j \in z}$ con respecto a los operadores de traslación y dilatación $\left(T_{h} f(x)=f(x-h), J_{s} f(x)=f\left(2^{s} x\right)\right)$. Además, se tienen $\left(4^{\prime}\right)\left(5^{\prime}\right)$ y $\left(6^{\prime}\right)$.

$4^{\prime} . V_{j}=J_{-j}\left(V_{0}\right)$ para toda $j \in \mathrm{Z}$

$5^{\prime} . V_{0}=T_{n}\left(V_{0}\right)$ para toda $n \in \mathbf{Z}$

6'. Para cada $j \in \mathrm{Z}$ el sistema $\left\{2^{j / 2} \phi_{j, k}\left(2^{j / 2} x-k\right)\right\}_{j, k \in Z}$ es una base ortonormal en $V_{j}$.

El análisis multirresolución conduce de una forma natural a la construcción de wavelets en 
términos de detalles que complementan la aproximación entre $V_{j}$ y $V_{j+1}$.

Por lo anterior, se pueden adoptar dos formas de ver un análisis multirresolución:

1. Tomando los sub-espacios $\left(V_{j}\right)_{j \in Z}$ como una base. Ellos tienen que satisfacer las condiciones (1)-(5), que por lo general son fáciles de comprobar. Entonces, se necesita encontrar una función escala que cumpla la condición (6). Esto generalmente no es tan evidente.

2. Partiendo de la función $\phi$ r se define $V_{0}$ como gen $\{\phi(t-m)\}_{m \in Z}$ y los demás espacios $V_{j}$ se definen por la condición (4) o (4'). La condición (5) se cumple automáticamente, y se tienen que comprobar las condiciones (1)-(3) y (6).

Se adopta en esta investigación la aplicación de un AMR utilizando el camino 2.

\section{Función spline}

Una función spline está formada por varios polinomios, Kincaid \& Cheney (1989). Cada uno es definido sobre un sub-intervalo, y se unen entre sí obedeciendo a ciertas condiciones de continuidad. Al fijar un número entero $k \geq 0$, y $n+1$ puntos (nodos) $t_{1}<t_{2}<\ldots<t_{n}$, una función $S(x)$ que satisface las condiciones:

1. En cada intervalo $\left[t_{i-1}, t_{i}\right), S(x)$ es un polinomio de grado $\leq k$.

2. $S(x)$ tiene una derivada de orden $(k-1)$ continua en $\left[t_{0}, t_{n}\right]$.

3. Se denomina una función spline de grado $k ; S(x)$ es, por tanto, un polinomio continuo por tramos de, cuando más, grado $k$, que tiene derivadas continuas de orden hasta $(k-1)$.

Entre los diferentes spline, la función spline cúbica es la más común y se define como la ecuación (32).
$y=S(x)=A_{k}\left(x-x_{k}\right)+B_{k}\left(x-x_{k}\right)^{2}+C_{k}\left(x-x_{k}\right)^{3}+D_{k}$

Donde las constantes $A_{k}, B_{k}, C_{k}, D_{k}$, son los coeficientes.

\section{Los B-splines}

Es un sistema de funciones spline simétricas y en forma de campana, a partir de las cuales se obtienen, mediante combinaciones lineales, todas las demás funciones spline. Son centrados, simétricos y de soporte compacto; Unser \& Aldroubi, (1993), Unser (1996). Los de Schoenberg se definen a partir de la ecuación (33).

$$
\beta^{n}(t)=\frac{1}{n !} \sum_{k=0}^{n+1}\left(\begin{array}{c}
n+1 \\
k
\end{array}\right)(-1)^{k}\left(t-k+\frac{n+1}{2}\right)^{n} \mu\left(t-k+\frac{n+1}{2}\right)
$$

En Unser (1996); Antonini, Barlaud, Mathieu \& Daubechies (1992); Unser \& Aldroubi (1993); y López \& De Armas (2010), el B-spline central de orden $n$ se define mediante la ecuación (34).

$\beta^{n}(t)=\frac{1}{n !} \sum_{k=0}^{n+1}\left(\begin{array}{c}n+1 \\ k\end{array}\right)(-1)^{k}\left(t-k+\frac{n+1}{2}\right)^{n} \mu\left(t-k+\frac{n+1}{2}\right)$

Cada B-spline de orden $n$ se puede construir por convoluciones sucesivas (ecuación (35)).

$\beta^{n}(t)=\underbrace{\beta^{0}(t) * \ldots * \beta^{0}(t)}_{(n+1) \text { veces }} ; \beta^{0}(t)=\left\{\begin{array}{l}1, \text { si } t \in\left[-\frac{1}{2}, \frac{1}{2}\right), \\ 0, \text { en otro caso }\end{array}\right\}$

$t \in \mathrm{R}, \mu$ es la función escalón unitario y $n$ es el orden de los polinomios.

Esta forma de construir recursivamente cada B-spline de orden $n$ puede hacerse a partir de un B-spline desplazado de orden $n-1$. Así, el spline cúbico se genera por la ecuación (36).

$$
\beta^{3}(t)=\beta^{0}(t) * \beta^{0}(t) * \beta^{0}(t) * \beta^{0}(t)
$$


Tomando:

$$
\phi(t)=\beta^{3}(t)=\left\{\begin{array}{ccc}
0 . & \text { si } & t<-2 \\
\frac{1}{6(2+t)^{3}}, & \text { si } & -2 \leq t \leq-1 \\
1+t-\frac{1}{6} t^{-}-\frac{1}{3}(1+t)^{3} & \text { si } & -1<t \leq 0 \\
1-t+\frac{1}{6} t^{3}-\frac{1}{3}(1-t)^{3} & \text { si } & 0<t \leq 1 \\
\frac{1}{6}(2-t)^{3} & \text { si } & 1<t \leq 2 \\
0 & \text { si } & t>2
\end{array}\right.
$$

La función escalada $\Phi(t)$ se diseña tomando la suma promedio de los B-splines x con el filtro identidad $\delta_{0}(k)$ o de Kronecker (ecuación (37)).

$$
\phi(t)=\sum_{k=-\infty} \delta_{0}(k) \beta^{3}(t-k)
$$

Considerando ahora el espacio $V_{j}$, formado por las funciones spline, donde cada polinomio definido sobre el intervalo $\left[k 2^{j} ;(k+1) 2^{j}\right]$, con $k \in Z$, es de grado tres:

$$
V_{j}=\left\{s(t): s(t)=\sum_{k=-\infty}^{\infty} c_{j}(k) \phi\left(2^{-j} t-k\right), t \in \mathrm{R}, c_{j} \in l_{2}\right\},
$$

$l_{2}$ es el espacio de las sucesiones cuadrado sumables $c(k), k \in Z$. $V_{j}$ es el conjunto de funciones spline cuadrado integrables de una dimensión, es decir, pertenecen a $L_{2}(R)$. Estas funciones son continuas de clase $C^{2}$.

Esta sucesión de espacios anidados $V_{j}$ y la función escalada $\phi(t)=\beta^{3}(t)$ definen un análisis multirresolución. La relación de escala viene dada mediante la ecuación (38); Unser, Aldroubi \& E. Murray (1993) y López \& De Armas (2010):

$$
\beta^{3}\left(\frac{t}{2}\right)=\sum_{k=-\infty}^{\infty} \mu_{2}^{3}(k) \beta^{3}(t-k)
$$

El filtro binomial Kernel $\mu_{2}^{3}(k)$ se conoce como filtro de impulso de respuesta finita o FIR, definido como:

$$
\mu_{2}^{3}(k)=\left\{\begin{array}{c}
0 \text { si } k<-2 \\
\frac{1}{8} \text { si } k=-2 \\
\frac{1}{2} \text { si } k=-1 \\
\frac{3}{4} \text { si } k=0 \\
\frac{1}{2} \text { si } k=1 \\
\frac{1}{8} \text { si } k=2 \\
0 \text { si } k \geq 2
\end{array}\right.
$$

La wavelet spline $\Psi\left(\frac{t}{2}\right)$ se construye al tomar la suma promedio de los B-splines cúbicos con una sucesión $w(k)$ definida a partir de la ecuación (39).

$$
\psi\left(\frac{t}{2}\right)=\sum_{k=-\infty}^{\infty} w(k) \beta^{3}(t-k)
$$

$\psi\left(\frac{t}{2}\right)$ es ortogonal al conjunto de B-splines expandidos $\left\{\beta^{3}\left(\frac{t}{2}-k\right)\right\}$.

Para conseguir una fórmula explícita de la wavelet B-spline de orden tres, se toma la ecuación (38) junto con la propiedad $\left\langle\beta^{3}(t), \beta^{3}(t-y)\right\rangle=\beta^{7}(y)$, consecuencia de la propiedad ya dada de convolución sucesiva. Finalmente, tiene la forma de la ecuación (40).

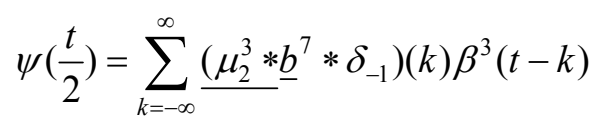

Y, cambiando $t$ por $2 t$ queda la ecuación (41).

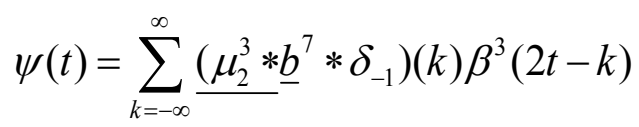

Desarrollando las operaciones, se tiene explícitamente la wavelet de medida nula, oscilante, de energía finita, bien localizada en un intervalo finito y con desvanecimiento en el tiempo; López \& De Armas (2010). 


\section{INVERSIÓN DE RADON UTILIZANDO WAVELETS}

Las wavelets y sus deformaciones, al estilo de Bonani, Durand \& Weiss (1996), se usan ahora para invertir la transformada de Radon (Berenstein \& Walnut (1994 y 1995), Rashid-Farrokhi, Liu, (1994); Natterer (2001); Walker (2008), Delaney \& Bresler (1995) o Destefano \& Olson (1994), Berenstein, \& Walnut (1997); Yin, Brian, Ferguson \& Abbott (2009); Ramm \& Zaslavsky (1993)). Como todas las wavelets tienen al menos un momento de desvanecimiento, esto sugiere que la transformada wavelet de $f$ pueda recuperarse localmente desde proyecciones locales.

En esta sección se presentan los teoremas que permiten invertir la transformada de Radon mediante la utilización de wavelets.

Proposición 7.1. Dada una wavelet $n$-dimensional separable $n>1$, como en la ecuación (42)

$$
\Psi(\mathbf{x})=\psi^{1}\left(x_{1}\right) \psi^{2}\left(x_{2}\right) \cdots \psi^{n}\left(x_{n}\right)
$$

donde la $\psi^{i}(t)$ satisface $\left|\hat{\psi}^{i}(\gamma)\right| \leq C_{i}(1+|\gamma|)^{-1}$ para todo $\gamma$, se define la familia de funciones unidimensional $\left\{\rho_{\theta}\right\}_{\theta \in S^{n \Perp 1}}$ por la ecuación (43)

$$
\hat{\rho}_{\theta}(\gamma)=\frac{1}{2}|\gamma|^{n-1} \hat{\psi}^{1}\left(\gamma \theta_{1}\right) \hat{\psi}^{2}\left(\gamma \theta_{2}\right) \ldots \hat{\psi}^{n}\left(\gamma \theta_{n}\right)
$$

donde $\theta=\left(\theta_{1}, \theta_{2}, \ldots, \theta_{n}\right) \in S^{n-1}$. Entonces para toda $f \in L^{1} \cap L^{2}\left(\mathrm{R}^{n}\right)$ se tiene la ecuación (44).

$$
\left(W_{\psi} f\right)(a, \mathbf{b})=a^{(1-n) / 2} \int_{S^{n \perp 1}}\left(W_{\rho \theta} \Re_{\theta} f\right)(a, b \cdot \theta) d \theta
$$

El punto central de esta proposición es observar que la transformada wavelet de una función $f(x)$ con cualquier wavelet madre y en cualquier escala y localización puede obtenerse por retroproyección de la transformada wavelet de la transformada de Radon de $f$, usando wavelets que varían con cada ángulo, pero que son admisibles para cada ángulo. La ecuación (43) se sigue inmediatamente de la ecuación de retroproyección filtrada (15) con una apropiada elección de $\Theta_{q}$ y observando que la dilatación conmuta con la retroproyección.

Proposición 7.2. Dada una wavelet unidimensional $\rho(t)$ tal que $\rho(t)=\rho(-t), \rho(t) \in \mathrm{R}$ para todo $t$ y la condición que se expresa en la ecuación (45), se define la función radial $\psi(x)$ mediante la ecuación (46).

$$
\begin{gathered}
\int_{0}^{\infty} \frac{|\hat{\rho}(r)|^{2}}{r^{2 n-1}} d r<\infty \\
\hat{\psi}(\xi)=2|\xi|^{1-n} \hat{\rho}(|\xi|)
\end{gathered}
$$

Entonces obtenemos la ecuación (47):

$$
\left(W_{\psi} f\right)(a, \mathbf{b})=a^{(1-n) / 2} \int_{S^{n \perp \perp}}\left(W_{\rho} \Re_{\theta} f\right)(a, b \cdot \theta) d \theta
$$

Esta proposición es un caso particular de la proposición 7.1, excepto que la wavelet unidimensional $\rho$ se fija con anterioridad para todos los ángulos $\theta$. Esto determina la wavelet bidimensional $\psi$. En la proposición 7.1, $\psi$ fue fijada con anterioridad determinando $\left\{\rho_{\theta}\right\}_{\theta \in[0,2 \pi]}$ para cada $\theta$.

En tomografía local se busca $\rho$ con soporte pequeño y muchos momentos de desvanecimiento. En este caso $\psi$ tendrá esencialmente el mismo radio de soporte que $\rho$. Por tanto, de la ecuación (47) los coeficientes wavelet de $f$ pueden recuperarse localmente desde las medidas locales de su transformada de Radon. Para los propósitos del procesamiento de imágenes, la idea es fijar la wavelet bidimensional $\psi$ apropiadamente, de acuerdo con la tarea deseada, y luego considerar las propiedades de $\rho_{\theta}$ para cada $\theta$.

Una vez calculada la transformada de la función $f(x)$, ahora se puede recuperar la función original $f(x)$ utilizando fórmulas estándar de inversión para la transformada wavelet continua.

Proposición 7.3. Dada $\rho(t)$ wavelet unidimensional que satisface $\rho(t)=\rho(-t), \rho(t) \in \mathrm{R}$ para todo $t$ y con $\lim _{\gamma \rightarrow 0}|\gamma|^{-1} \hat{\rho}(\gamma)=\frac{1}{2}$. Se define la fun-

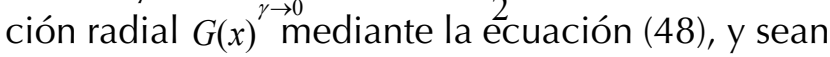
$\psi^{1}(x), \psi^{2}(x)$ como en la ecuación (46) y la (47). 
Entonces la transformada wavelet de $f(x)$ se expresa mediante las ecuaciones (49) y (50).

$$
\begin{gathered}
\hat{G}(\xi)=2|\xi|^{-1} \hat{\rho}(|\xi|) \\
\left(W_{\psi^{\prime}} f\right)\left(2^{j}, x\right)=2^{j / 2} \int_{0}^{2 \pi} \cos \theta\left(W_{\frac{d \rho}{d t}} \Re_{\theta} f\right)\left(2^{j}, x \cdot \theta\right) d \theta \\
\left(W_{\psi^{2}} f\right)\left(2^{j}, x\right)=2^{j / 2} \int_{0}^{2 \pi} \sin \theta\left(W_{\frac{d \rho}{d t}} \Re_{\theta} f\right)\left(2^{j}, x \cdot \theta\right) d \theta
\end{gathered}
$$

Proposición 7.4. Sea $\rho(t)$ y $G(x)$ que cumplen las condiciones del teorema anterior, y sea $\beta \in[0,2 \pi]$. Entonces la transformada de $f(x)$ se puede escribir como en la ecuación (51).

$\left(W_{\psi^{\beta}} f\right)\left(2^{j}, x\right)=2^{j / 2} \int_{0}^{2 \pi} \cos (\beta-\theta)\left(W_{\frac{d p}{d t}} \Re_{\theta} f\right)\left(2^{j}, x \cdot \theta\right) d \theta(51)$

Los coeficientes se obtienen a partir de los productos interiores definidos en las ecuaciones (52)-(55).

$$
\begin{aligned}
& \left\langle f, \Phi_{j, k}\right\rangle=2^{-j / 2} \int_{0}^{2 \pi}\left(W_{\sigma \theta} \Re_{\theta} f\right)\left(2^{j}, 2^{j}(k \cdot \theta)\right) d \theta \\
& \left\langle f, \Psi_{j, k}^{i}\right\rangle=2^{-j / 2} \int_{0}^{2 \pi}\left(W_{\rho_{\theta}^{i}} \Re_{\theta} f\right)\left(2^{j}, 2^{j}(k \cdot \theta)\right) d \theta \\
& \left\langle f, \Phi_{j, k}\right\rangle=2^{-j / 2} \int_{0}^{2 \pi}\left(W_{\sigma_{\theta}} \Re_{\theta} f\right)\left(2^{j}, 2^{j}(k \cdot \theta)\right) d \theta \\
& \left\langle f, \Psi_{j, k}^{i}\right\rangle=2^{-j / 2} \int_{0}^{2 \pi}\left(W_{\rho_{\theta}^{i}} \mathfrak{R}_{\theta} f\right)\left(2^{j}, 2^{j}(k \cdot \theta)\right) d \theta
\end{aligned}
$$

Y se expresan en términos de la transformada wavelet de las proyecciones para cada ángulo, donde se definen los siguientes filtros en términos de la transformada de Fourier (ecuaciones (56)-(59)).

$$
\begin{aligned}
& \hat{\sigma}_{\theta}(\gamma)=\frac{1}{2}|\gamma| \hat{\varphi}(\gamma \cos \theta) \hat{\varphi}(\gamma \sin \theta) \\
& \hat{\sigma}_{\theta}^{1}(\gamma)=\frac{1}{2}|\gamma| \hat{\varphi}(\gamma \cos \theta) \hat{\varphi}(\gamma \sin \theta) \\
& \hat{\sigma}_{\theta}^{2}(\gamma)=\frac{1}{2}|\gamma| \hat{\varphi}(\gamma \cos \theta) \hat{\varphi}(\gamma \sin \theta) \\
& \hat{\sigma}_{\theta}^{3}(\gamma)=\frac{1}{2}|\gamma| \hat{\varphi}(\gamma \cos \theta) \hat{\varphi}(\gamma \sin \theta)
\end{aligned}
$$

Y los filtros duales, definidos igualmente en Berenstein \& Walnut (1994 y 1995) en términos de la transformada de Fourier de las duales (ecuaciones (60)-(63)), y que mediante la transformada de Hilbert y la transformada de la derivada se obtiene $\rho_{\theta}^{i}: i=1,2,3$; como se muestra en la figura 1 .

$$
\begin{aligned}
& \hat{\dot{\sigma}}_{\theta}(\gamma)=\frac{1}{2}|\gamma| \hat{\dot{\varphi}}(\gamma \cos \theta) \hat{\dot{\varphi}}(\gamma \sin \theta) \\
& \hat{\dot{\sigma}}_{\theta}^{1}(\gamma)=\frac{1}{2}|\gamma| \hat{\dot{\varphi}}(\gamma \cos \theta) \hat{\dot{\varphi}}(\gamma \sin \theta) \\
& \hat{\sigma}_{\theta}^{2}(\gamma)=\frac{1}{2}|\gamma| \hat{\dot{\varphi}}(\gamma \cos \theta) \hat{\dot{\varphi}}(\gamma \sin \theta) \\
& \hat{\dot{\sigma}}_{\theta}^{3}(\gamma)=\frac{1}{2}|\gamma| \hat{\dot{\varphi}}(\gamma \cos \theta) \hat{\dot{\varphi}}(\gamma \sin \theta)
\end{aligned}
$$

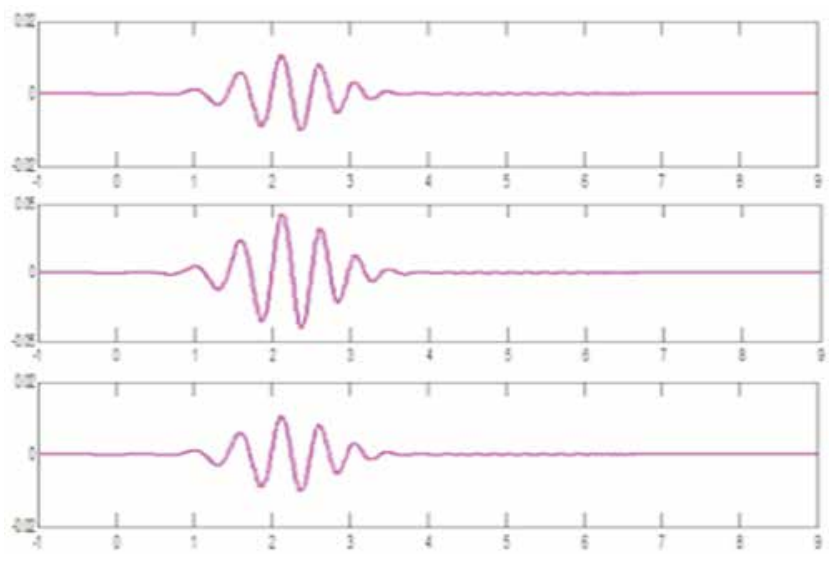

Figura 1. $\rho^{1}$ proyección 10, 45 y 90 grados.

Fuente: Elaboración propia.

\section{Inversión con wavelets separables}

Una imagen $f(\mathbf{x}), \quad \mathbf{x} \in \mathrm{R}^{2}$ de acuerdo con Berenstein \& Walnut (1994 y 1995), puede expresarse mediante la transformada wavelet a partir de la ecuación (64) o como las ecuaciones (65) y (66).

$$
\begin{aligned}
& f(\mathbf{x})=\sum_{j, k} \sum_{i=1}^{3}\left\langle f, \Psi_{j, k}^{i}\right\rangle \Psi_{j, k}^{i}=\sum_{j, k} \sum_{i=1}^{3}\left\langle f, \Psi_{j, k}^{i}\right\rangle \Psi_{j, k}^{i} \\
& f(\mathbf{x})=\sum_{k \in Z^{2}}\left\langle f, \Phi_{j, k}\right\rangle \Phi_{j, k}+\sum_{j=-\infty}^{J} \sum_{k \in Z^{2}} \sum_{i=1}^{3}\left\langle f, \Psi_{j, k}^{i}\right\rangle \Psi_{j, k}^{i} \\
& f(\mathbf{x})=\sum_{k \in Z^{2}}\left\langle f, \Phi_{j, k}\right\rangle \dot{\Phi}_{j, k}+\sum_{j=-\infty}^{J} \sum_{k \in Z^{2}} \sum_{i=1}^{3}\left\langle f, \Psi_{j, k}^{i}\right\rangle \Psi_{j, k}^{i}
\end{aligned}
$$


Donde

$$
\begin{gathered}
\Phi_{j, k}(\mathbf{x})=\varphi_{j, k_{1}}\left(x_{1}\right) \varphi_{j, k_{2}}\left(x_{2}\right) \\
\Psi_{j, k}^{1}(\mathbf{x})=\varphi_{j, k_{1}}\left(x_{1}\right) \psi_{j, k_{2}}\left(x_{2}\right) \\
\Psi_{j, k}^{2}(\mathbf{x})=\psi_{j, k_{1}}\left(x_{1}\right) \varphi_{j, k_{2}}\left(x_{2}\right) \\
\Psi_{j, k}^{3}(\mathbf{x})=\psi_{j, k_{1}}\left(x_{1}\right) \psi_{j, k_{2}}\left(x_{2}\right)
\end{gathered}
$$

$$
\begin{gathered}
\Phi(x)=\varphi\left(x_{1}\right) \varphi\left(x_{2}\right) \\
\Psi^{1}(x)=\varphi\left(x_{1}\right) \psi\left(x_{2}\right) \\
\Psi^{2}(x)=\psi\left(x_{1}\right) \varphi\left(x_{2}\right) \\
\Psi^{3}(x)=\psi\left(x_{1}\right) \psi\left(x_{2}\right)
\end{gathered}
$$

Las funciones duales respectivas, ecuaciones

Y (71)-(74)

$$
\begin{gathered}
\Phi_{j, k}(\mathbf{x})=\varphi_{j, k_{1}}\left(x_{1}\right) \varphi_{j, k_{2}}\left(x_{2}\right) \\
\Psi_{j, k}^{1}(\mathbf{x})=\varphi_{j, k_{1}}\left(x_{1}\right) \psi_{j, k_{2}}\left(x_{2}\right) \\
\Psi_{j, k}^{2}(\mathbf{x})=\psi_{j, k_{1}}\left(x_{1}\right) \varphi_{j, k_{2}}\left(x_{2}\right) \\
\Psi_{j, k}^{2}(\mathbf{x})=\psi_{i, k_{1}}\left(x_{1}\right) \psi_{i, k_{j}}\left(x_{2}\right)
\end{gathered}
$$

$$
\Phi(x)=\varphi\left(x_{1}\right) \varphi\left(x_{2}\right)
$$$$
\Psi^{1}(x)=\varphi\left(x_{1}\right) \psi\left(x_{2}\right)
$$$$
\Psi^{2}(x)=\psi\left(x_{1}\right) \varphi\left(x_{2}\right)
$$$$
\Psi^{3}(x)=\psi\left(x_{1}\right) \psi\left(x_{2}\right)
$$

Correspondiendo a las bases wavelets separables biortogonales de las splines cúbicas $\varphi$ y $\psi$.

En la figura 2 se muestra la función escalada y wavelet.

diente wavelet separable bidimensional es la formación de las ecuaciones (67)-(70).
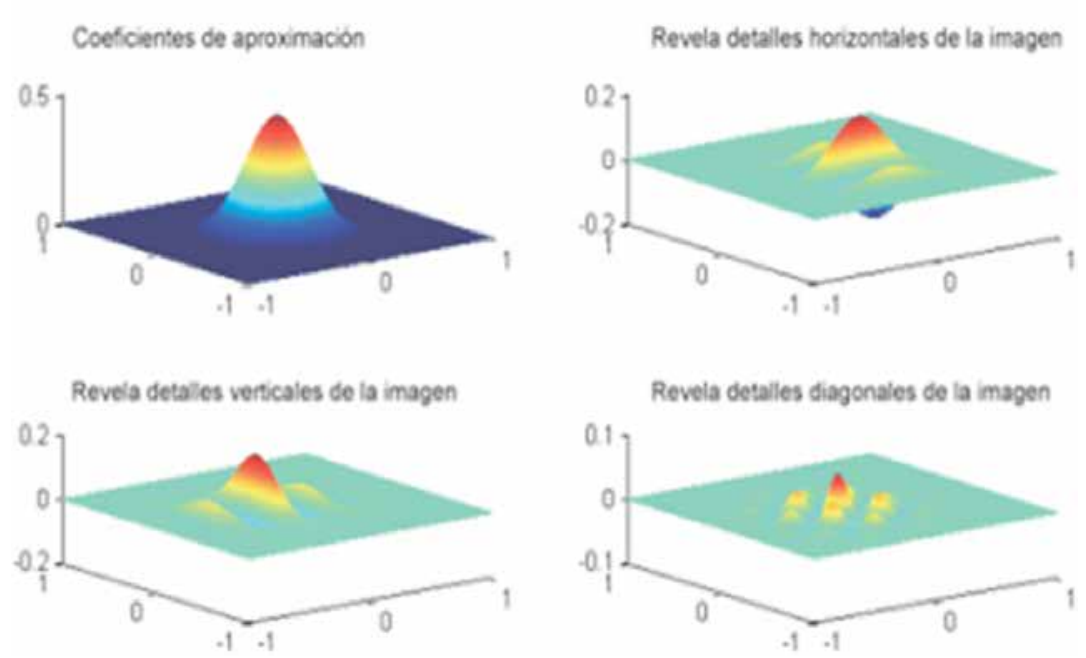

Figura 2. Las funciones escalada y wavelet separables, para la función $f(\mathbf{x}), \mathbf{x} \in \mathrm{R}^{2}: \psi^{1}(x, y)$ (detalles horizontales). $\psi^{2}(x, y)$ (detalles verticales); $\psi^{3}(x, y)$ (detalles diagonales).

Fuente: Elaboración propia. 


\section{ANÁLISIS Y RESULTADOS}

En el algoritmo de reconstrucción que proponen los autores en Berenstein \& Walnut (1994), basado en trabajos realizados sobre la localización de la transformada de Radon, las wavelets utilizadas son Daubechis de orden 4, 6, y 10. En el presente trabajo se ha acordado escoger la wavelet biortogonal spline cúbica con soporte compacto. Para comprobar la reconstrucción de una imagen cuya función inicial $\psi$ es elegida como una wavelet de este tipo, entonces cada función o imagen de energía finita resulta ser una superposición de tales componentes multiplicadas por un coeficiente. Esta representación de la señal es única y conserva en los coeficientes la energía de la misma.

La imagen original tiene un tamaño de 512 x 512 pixeles, y los datos de los bordes locales se calcularon usando solamente proyecciones que atravesaban la región de interés escogida (figura 3).

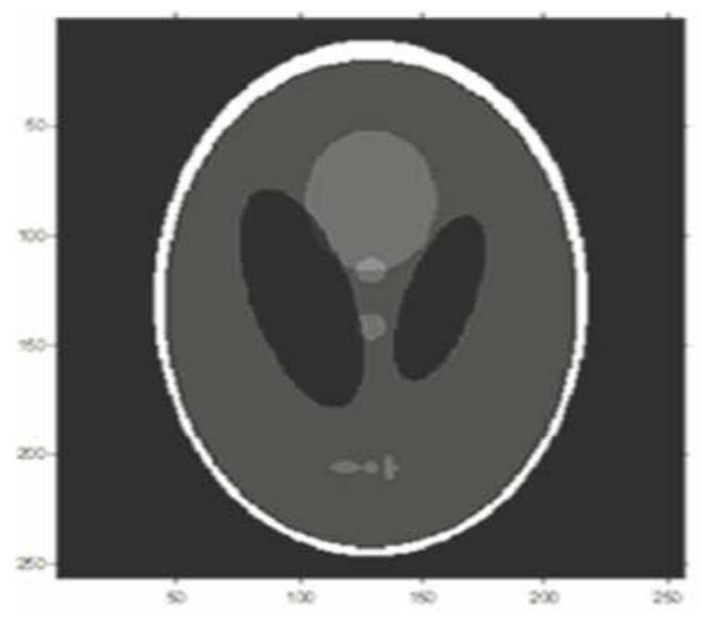

Figura 3. Fantasma de Shepp-Logan.

Fuente: Elaboración propia.
Se escoge una región de hasta 91 pixeles de diámetro localizada en el centro del fantasma de Shepp-Logan. La imagen fue reconstruida usando el algoritmo de transformada wavelet separable dada por la ecuación (66). Luego, se muestra la reconstrucción comparada con la reconstrucción estándar de retroproyección filtrada utilizando la transformada rápida de Fourier.

Como resultado, se ha implementado un algoritmo aprovechando las propiedades de las wavelets para localizar la transformada de Radon y usarla para reconstruir una región local. El algoritmo se basa en la observación intuitiva de que para algunas bases wavelet con momentos de desvanecimiento suficientemente numerosos, la versión rampa-filtrada de la función de escalado, así como la función wavelet tienen decaimiento rápido. Esto explica que un alto número de momentos de desvanecimiento puede contribuir al incremento en el decaimiento de transformada de Hilbert (Chaudhury \& Unser, 2011). Es decir, dada $\psi(x)$ una wavelet diferenciable con $n$ momentos de desvanecimiento, $H \psi(x)$ está bien definida y tiene a su vez $n$ momentos de desvanecimiento. Así mismo la suavidad de $H \psi(x)$ se tiene al sacar provecho del dominio de Fourier de la transformación, y el hecho de que la suavidad de una función se relaciona con el decaimiento de su transformada de Fourier. En general, cuanto mejor sea el decaimiento de la transformada de Fourier, más suave es la función, y viceversa. Como la wavelet B-spline cúbica es una señal de energía finita y pertenece al espacio de Sobolev $W^{2, p}(\Omega)$ para todo $p<3+\frac{1}{2}$, esto explica la suavidad de la wavelet y su transformada de Hilbert utilizadas en el algoritmo diseñado, las cuales son dos veces continuamente diferenciables.

En la figura 4 se ilustra la región de interés, donde se puede variar la cantidad de pixeles del diámetro de la misma. 


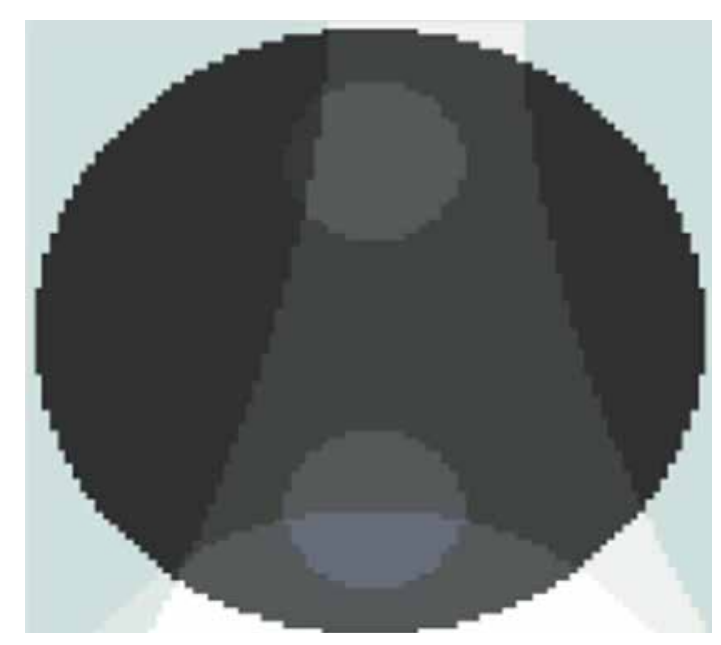

Figura 4. Región central del fantasma.

Fuente: Elaboración propia.

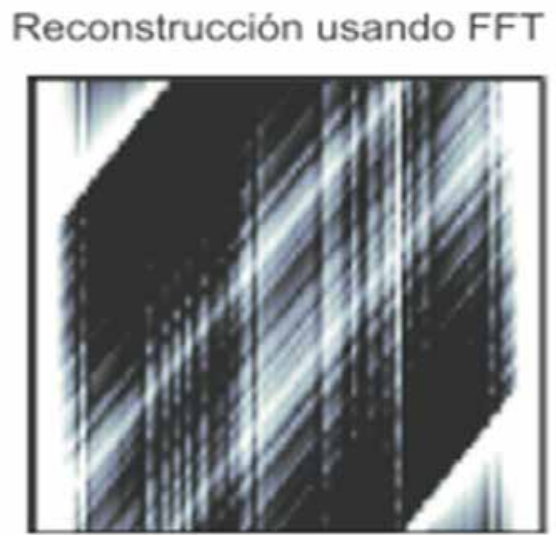

\section{Reconstrucción usando B-spline}

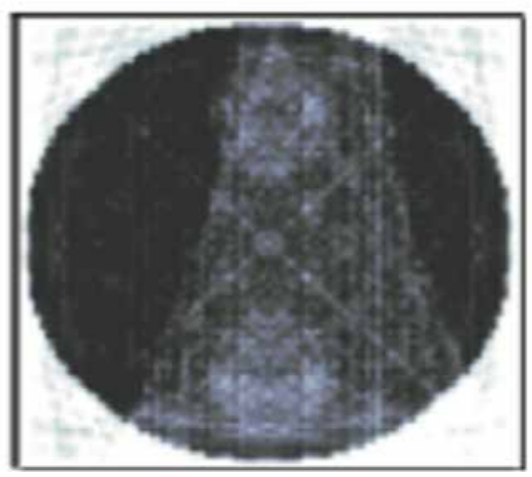

Figura 5. Reconstrucción utilizando 10 proyecciones.

Fuente: Elaboración propia.

En la figura 5 se contrasta el resultado de la reconstrucción obtenida del algoritmo propuesto frente a la utilización del método basado en FFT, utilizando 10 proyecciones.
En la figura 6 se utilizan 55 proyecciones, y en la figura 7 se utilizan 80 proyecciones. 

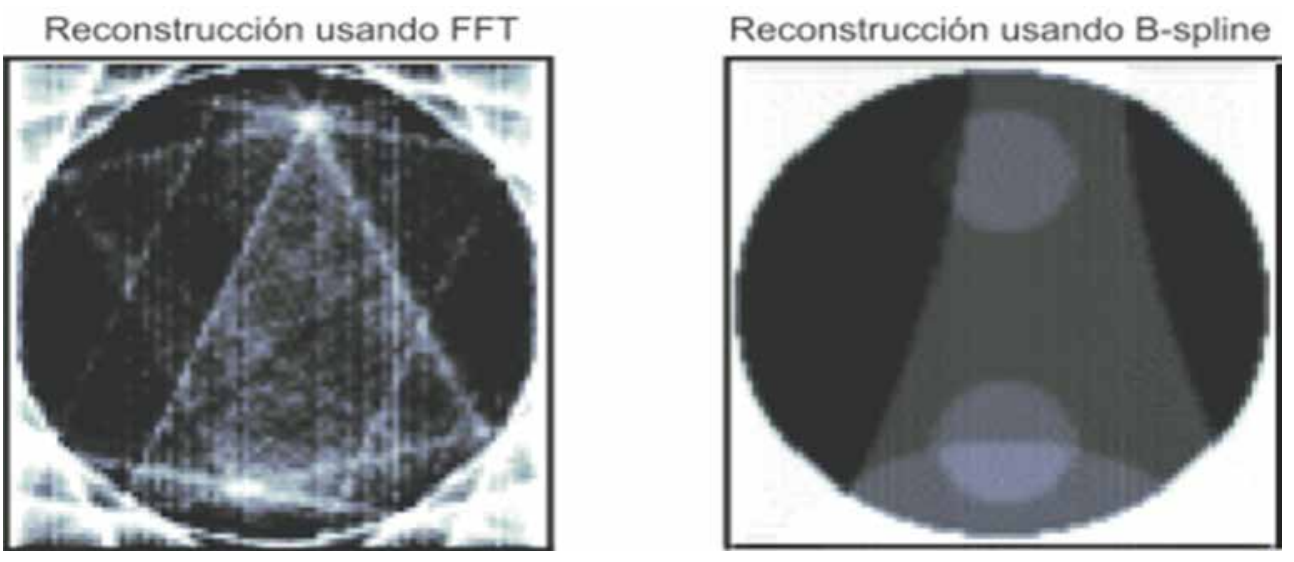

Figura 6. Reconstrucción utilizando 55 proyecciones.

Fuente: Elaboración propia.
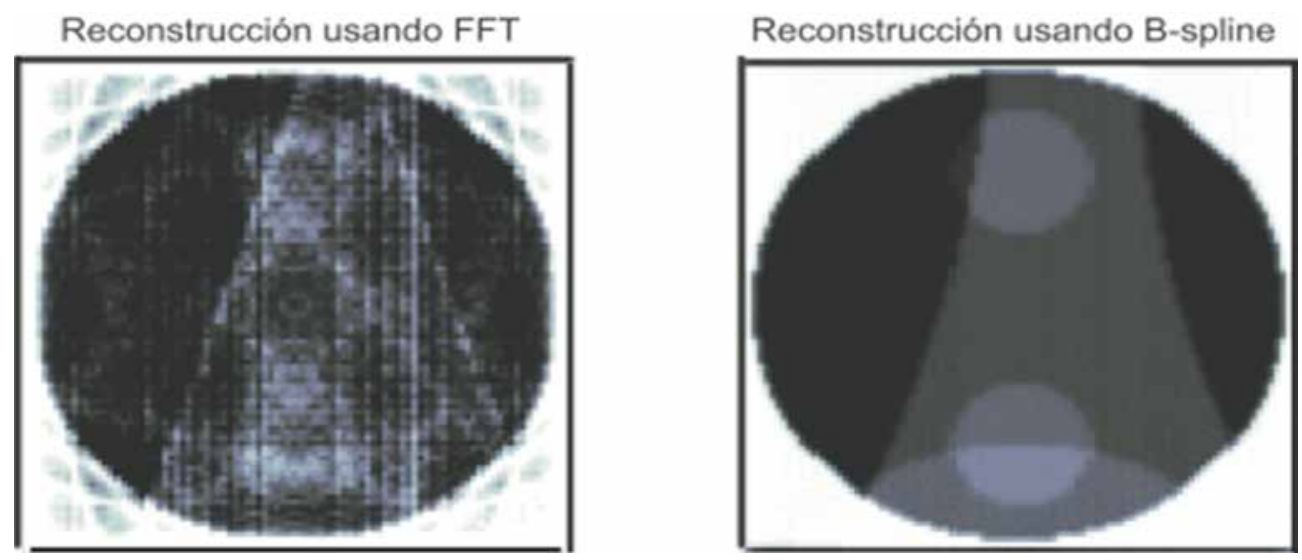

Figura 7. Reconstrucción utilizando 80 proyecciones.

Fuente: Elaboración propia.

De las anteriores gráficas se puede inferir que para reconstruir, incluso una pequeña $\mathrm{ROI}$, se consideran algunos datos fuera de ella; Natterer (2001). Siguiendo a F. Rashid-Farrokhi, Liu \& Berenstein (1995), y Rashid-Farrokhi, Liu \& Berenstein (1997); Yin, Ferguson \& Abbott (2009), los límites superiores del error en términos de la cantidad de tales datos no locales son mayores en el ilustrado en la reconstrucción estándar FFT (Brigham, 1989) que con la implementada con B-spline, debido a la calidad de la localización wavelet pues los soportes de la wavelet madre y los filtros rampa escalados se mantienen en errores relativos inferiores a $0.4 \%$ utilizando cerca de 6 pixeles fuera de la ROI. En consecuencia, la mayor parte de la energía de una imagen se compacta en la imagen aproximada, por lo que la estimación de error allí da una buena indicación del error global. 


\section{CONCLUSIONES}

La propuesta de un algoritmo cuyo reconstructor se diseña a partir de wavelets escaladas y wavelets madre de tipo B-spline cúbicos, al evidenciar claramente la eficiencia del mismo, es una elección plausible puesto que ellas son wavelets con soporte compacto continuamente diferenciables. Este es el caso de la wavelet spline cúbica escogida, que, además de ser continuamente diferenciable, tiene momentos de desvanecimiento y satisface condiciones de decaimiento. Se concluye que la importancia de este resultado es formalizar que, al exigir que $\psi(x)$ tenga un gran número de momentos de desvanecimiento, se puede efectivamente hacer $H \psi(x)$ tan localizada como $\psi(x)$ - Hasta ahora esto se había observado cualitativamente con la localización de la transformada wavelet de la transformada de Radon, Brigham (1989); Ramm \& Zaslavsky (1993) y Destefano \& Olson (1994). Teniendo en cuenta el caso especial de $\psi(x)$ wavelet B-spline de grado $n-1$, entonces $H \psi(x)$ vuelve a ser una wavelet B-spline fraccional del mismo grado, y por consiguiente tiene el mismo decaimiento; Unser \& Aldroubi (1993); Chaudhury \& Unser (2011). Por lo anterior, se garantiza la predicción de la reconstrucción propuesta, mejorando la basada en FFT, ya que se sabe que $\psi(x)$ tiene $n$ momentos de desvanecimiento. El método mostrado utiliza las propiedades de las wavelets B-spline cúbicas para localizar la transformada de Radon y poder reconstruir eficientemente una región local del fantasma de Shepp Logan, a partir de datos digitales.

Las wavelets pueden aplicarse con ventaja sobre otros métodos basados en Fourier, como se puede observar en las múltiples aplicaciones: Walnut(1992); Clarke (1995); Donoho (1993); Shapiro (1993); Quak, Weyrich (1994); Shen \& Galerkin (2000); Tang \& Yang (2000); Tao, Mang \& Yuesheng (2000); Stark (2005), pues el procesamiento de imágenes es más eficiente por efecto de la capacidad de localización.
Como perspectiva de investigación, además de la utilidad sistemática en tomografía, actualmente se utilizan las wavelets en la investigación de materiales. En este sentido Lebon, Rodríguez-Ramos, López-Realpozo, Bravo-Castillero, Guinovart-Díaz \& Mesejo (2006), por ejemplo, estudian teóricamente problemas unidimensionales de homogenización, en particular el caso de la determinación de propiedades efectivas de compuestos laminados con características especiales, solucionando numéricamente las ecuaciones resultantes con wavelets de soporte compacto y análisis multirresolución.

\section{FINANCIAMIENTO}

\section{Universidad EAFIT}

\section{REFERENCIAS}

Aldroubi, A. (1996). The wavelet transform: A surfing guide. Wavelets in Medicine and Biology. A. Audroubi, M. Unser (eds.), pp. 3-36. New York: CRC Press.

Antonini, M.; Barlaud, P.; Mathieu, P.; Daubechies, I. (1992). Image Coding Using Wavelet Transform. IEEE Trans. Image Proc., vol. 1(2), pp.205-220.

Berenstein, C.; Walnut, D. (1994). Local inversion of the Radon transform in even dimensions using Wavelets. S. Gindikin and P. Michor, eds., 75 years of Radon Transform, pp. 45-69. Cambridge, MA.: International Press Co., Ltd.

Berenstein, C.; Walnut, D. (1995.). Wavelets and local tomography. Wavelets in Medicine and Biology. CRC Press.

Brigham, E. (1988). The Fast Fourier Transform and its Applications. Ann Arbor, Michigan: Prentice Hall.

Boggess, A.; Narcowich, F. (2007). A First Course in Wavelets with Fourier analysis. Ann Arbor, Michigan: Prentice Hall.

Bonani, A.; Durand, S.; Weiss, G. Wavelets obtained by continuos deformations of the Haar wavelet. Revista Mat. Iberoamericana, vol. 12(1). 
Chaudhury, K.; Unser, M. (April 2011). On the Hilbert Transform of Wavelets. IEEE, Transactions on Signal Processing, vol. 59(4).

Chui, C. (1992). An Introduction to Wavelets. New York: Academic Press.

Clarke, R. (1985). Transform Coding of Images. San Diego, CA: Academic Press.

Clarke, R. (1995). Digital Compression of Still Images and Video. San Diego, CA: Academic Press.

Daubechies, I. (1990). The wavelet transform, time-frequency localization and signal analysis. IEEE Transactions on Information. Theory, vol. 36(11), pp. 961-1005.

Daubechies, I. (1992). Ten Lectures on Wavelets. Philadelphia: CBMS Series 61, SIAM.

Daubechies, I. (Aug. 2006). Orthonormal bases of compactly supported wavelets. Communications on pure and Applied Mathematics, vol. 41(7), pp. 909-996.

Delaney, A.; Bresler, Y. (June 1995). Multiresolution tomographic reconstruction using wavelets. IEEE Transactions on Image Processing, vol. 4 (3).

Destefano, J.; Olson, T. (Aug. 1994). Wavelet Localization of the Radon Transform. IEEE Transactions on Signal Processing, vol. 42 (8), pp. 2055-2067.

Donoho, D. (1993). Nonlinear Wavelet Methods for Recovery of Signals, Densities and Spectra from Indirect and Noyse Data. Different Perspectives on Wavelets. Proceedings of Symposia in Pure Math., AMS., I. Daubechies, Edt., 47, pp. 173-205.

Faridani, A.; Keinert, F.; Natterer, F.; Ritman, E.; Smith, K. (1990). Local and global tomography. New York: Springer-Verlag.

Faridani, A.; Ritman, E.; Smith, K. (1993). Local Tomography. SIAM Journal on Applied Mathematics, vol. 52(2), pp. 459-484.

Faridani, A.; Finch, D.; Ritman, E.; Smith, K. (Aug. 1997). Local Tomography II. SIAM Journal on Applied Mathematics, vol. 57( 4), pp. 1095-1127.

Folland, G. (1999). Real Analysis. New York: John Wiley \& Sons, Inc, 2nd edition.

Guédon, J.; Unser, M. (1992). Least squares and spline filtered back-projection. NCRR Rep. 52/92, Nat. Inst. Health.
Goswami, J.; Chan, A. (1999). Fundamentals of Wavelets Theory, Algorithms, and Applications. John Wiley \& sons, Inc.

Helgason, S. (1999). The Radon Transform. Boston: Birkhäuser. Second Edition.

Hernández, E.; Weiss, G. (1996). A First Course on Wavelets. Boca Raton FL: CRC Press.

Hong, D.; Wang, J.; Gardner, R. (2005). Real Analysis with an Introduction to Wavelets and Applications. Academic Press, Elsevier.

Irino, T.; Kawahara, H. (1993). Signal reconstruction from modifed auditory wavelet transform. IEEE Trans. Signal Process, vol. 41(32), pp. 3549-3553.

Kincaid, D.; Cheney, W. (1994). Análisis Numérico. Edit. E.U.A. pp. 323-363.

Lebon, F.; Rodríguez-Ramos, R.; López-Realpozo, J.C.; Bravo-Castillero, J.; Guinovart-Díaz, R.; Mesejo, A. (January 2006). Effective Properties of Nonlinear Laminated Composites With Perfect Adhesion. Transactions of the ASME, vol. 73, pp. 174-178.

López, R.; De Armas, R. (2010). Algoritmo para detectar eventos epilépticos a partir de wavelets analizando la función de energía. Tesis de maestría en matemáticas aplicadas. Universidad EAFIT, Medellín.

Maass, P. (agosto 1992). The interior Radon Transform. SIAM Journal on Applied Mathematics, vol. 52(3), pp. 710-724.

Mallat, S. (1989). A theory for multiresolution signal decomposition: The wavelet representation. IEEE Trans. Pattern Anal. Mach. Intell, vol. 11(33), pp. 674-693.

Mallat, S. (1989). Multiresolution approximations and wavelet orthonormal bases for $\mathrm{L}_{2}$. Trans. of Amer. Math. Soc. 315, pp. 69-87.

Mallat, S.; Hwang, W. (1992). Singularity detection and processing with wavelets. IEEE Trans. Inform. Theory (Special Issue on Wavelet Transforms and Multiresolution Signal Analysis), 38(2), pp. 617-643.

Mallat, S. (2008). Wavelet Tour of signal Processing. Boston: American Press.

Mertins, A. (1996). Signal Analysis Wavelets, Filter Banks, Time-Frequency Transforms and Applications. New York: John Wiley \& sons.

Meyer, Y. (1990). Ondelettes et operateurs, I: Ondelettes. Paris: Herman. 
Natterer, F. (2001). The Mathematics of Computarized Tomography. SIAM.

Natterer, F.; Wübbeling, F. (2001). Mathematical Methods in Image Reconstruction. SIAM.

Pinsky, M. (2001). Introduction to Fourier Analysis and wavelet. Brooks/Cole, NJ.

Quak, E.; Weyrich, N. (1994). Decomposition and reconstruction algorithms for spline wavelet on a bounded interval. Appl. and Comp. Harmonic Anal. (ACHA), vol. 1, pp. 217-231.

Ramm, A.; Zaslavsky, A. (1993). Reconstructing singularities of a function from its Radon transform. Math. and Comput. Modelling, vol. 18(1), pp. 109-138.

Ramm, A.; Zaslavsky, A. (1993). Singularities of the Radon transform. Bull. AMS, vol. 25(1), pp. 109-115.

Rashid-Farrokhi, F.; Liu, K.; Berenstein, C.; Walnut, D. (1995). Localized wavelet based computerized tomography. Washington: Proceedings ICIP-95.

Rashid-Farrokhi, F., Liu, R., K.J.; Berenstein, C.; Walnut, D. (Oct. 1997). Wavelet-Based Multiresolution Local Tomography. IEEE. Trans. On image Process, vol. 6(10), pp. 1412-1429.

Rioul, O.; Duhamel, P. (1992). Fast algorithms for discrete and continuous wavelet transform. IEEE Trans. Inform. Theory, vol. 38, pp. 569-586.

Shapiro, J. (1993). Embedded image coding using zerotrees of wavelet coeficients. IEEE Trans. Signal Proc., vol. 41, pp. 3445-3462.

Shen, X.; Galerkin, A. (2000). Wavelet method for a singular convolution equation on the real line. J. Int. Equa. Appl., vol. 12, pp. 157-176.

Stark, H. (2005). Wavelets and Signal Processing An Application-Based Introduction. Springer.

Tang, Y.; Yang, L. (2000). Wavelet Theory and Its Application to Pattern Recognition. World Scientific.
Tao, Q.; Mang, V.; Yuesheng, X. (2000). Wavelet Analysis and Applications. Berlin: Birkhäuser Verlag Basel.

Unser, M.; Aldroubi, A.; Murray, E. (1993). A family of polynomial spline wavelet transforms. Signal Processing, vol. 30, pp. 141-162.

Unser, M.; Aldroubi, A. (1993). B-spline processing I: Theory and II: Eficient design and application. IEEE Trans. Signal Process, vol. 41, pp. 821-848.

Unser, M. (1996). A practical guide to implementation of the wavelet transforms. Wavelets in Medicine and Biology. New York: CRC Press, pp. 37-76.

Walker, J. (2008). Local inversion of the Radon Transform in the plane using wavelets. A Primer on wavelets and Their Scientific Applications. Second edition. Chapman \& Hall/CRC. Taylor \& Francis Group.

Walnut, D. (1992). Application of Gabor and Wavelet expansions to the Radon Transform, Probabilistic and Stochastic Methods in Analysis, with applications. J. Byrnes et al. eds., Kluwer Academic Publishers, Inc., pp. 187-205.

Walnut, D. (1993). Local inversion of the Radon Transform in the plane using wavelets. San Diego: Proceedings of SPIE's. International Symposium on Optics, Imaging, and Instrumentation.

Walnut, D. (2002). An Introduction to Wavelet Analysis. Birkhäuser.

Walter, G.; Shen, X. (1999). Deconvolution using Meyer wavelets. J. Integral Equations Appl., vol. 11, pp. 515-534.

Wojtaszczyk, P. (1997). A Mathematical Introduction to Wavelets. Cambridge University Press.

Yin, X.; Ferguson, B.; Abbott, D. (2009). Wavelet based local tomographic image using terahertz techniques. Digital Signal Processing, vol. 19, pp.750763. Elsevier.

\section{(c) $(1) \Theta$}


